\title{
Exclusive live orthodontic education event
}

Optident, a Henry Schein company, will present a special full-day live education event to advance the future of orthodontics on 15 December 2021 at the Royal College of Physicians in London.

Dr Luis Carrière (pictured), who obtained his dental degree from the University of Complutense in Madrid, Spain, in 1991, attended the University of Barcelona to receive his Master of Science in Orthodontics in 1994, and in 2006, his Doctorate in Orthodontics, Cum Laude; and Dr Mario Chorak, who graduated at University of Kentucky College of Dentistry in the US with High Distinction in 1999, and earned his Certificate of Orthodontics and Dentofacial Orthopedics from the Oregon Health Sciences University in 2001, will present new paradigms in clear aligner therapy to an intimate audience - demonstrating to orthodontists how to achieve ideal clinical results, whilst offering patients accessibility, comfort, and building up valuable relationships along the journey.



Dr Carrière will discuss in detail his Minimum Touch Orthodontics approach, which is growing in popularity. In conjunction with the SAGITTAL FIRST philosophy, self-ligating brackets and aligners, Dr Carrière will simplify some of the cost complex cases using these techniques. Following this, Dr Chorak will present his combination cases using both the Carriere MOTION 3D APPLIANCE and Reveal Clear Aligners, from basic to advanced clinical cases.

Henry Schein's Reveal Clear Aligners (Reveal) is a convenient solution for patients looking to enhance their smile, with a treatment plan approved by a dentist of their choosing. The aligners are designed with patient comfort and aesthetics in mind and are clearer than most other aligners in the market. They reduce or eliminate the need for attachments in mild to moderate cases, featuring a smooth scalloped edge to enhance patient comfort. Henry Schein's Orthodontic Specialists provide in-practice support of the system and advice on how to incorporate aligner workflows into your treatment plans.

This course is open to all dental professionals with an interest in orthodontics. For more information on the course and registration visit: https:// www.eventbrite.co.uk/e/minimum-touchorthodontics-a-new-paradigm-in-clearaligner-therapy-tickets-189541783577.

\section{Facemasks that kill viruses}

Lancaster-based ViraCoat Ltd has submitted its FFP2 NR mask to rigorous testing to ISO18184 and EN149 compliance standards and gained certification that, within 30 minutes of

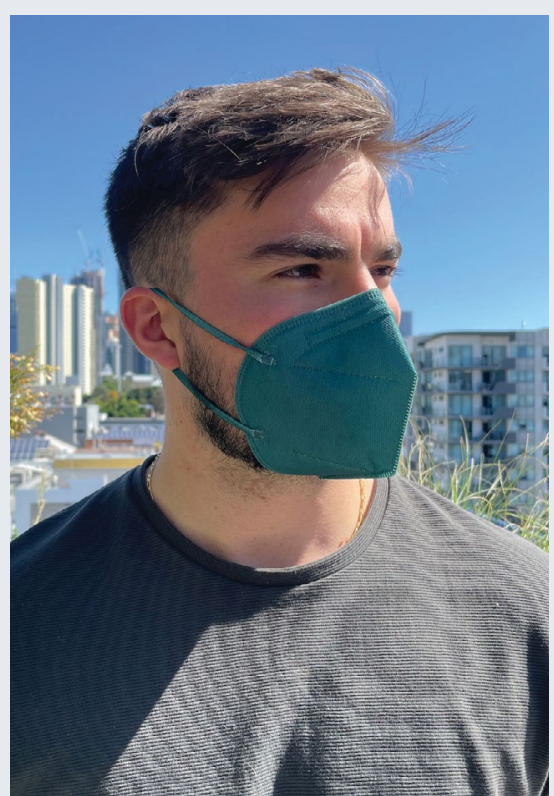

contact, the SARS-COV-2 virus that causes COVID-19 was inactivated. This confirms that the coating is virucidal, meaning that it begins to kill viruses on contact and destroys SARS-COV-2 so that it cannot enter host cells. It is also antiviral, which prevents viruses from replicating, and antimicrobial, which prevents bacteria from developing, thus providing additional protection to the mask wearer. As well as reducing transmission whilst being worn, the coating can significantly reduce the risk of contamination from handling the masks whilst putting them on, taking them off and disposing of them. Conventional masks only offer limited protection by filtering the air we breathe in and out, with considerable leakage around the edges. The ViraCoat masks are moulded and have an adjustable nose bridge for an optimal seal to prevent contamination by infectious aerosols - tiny virus particles that linger in the air and are now widely accepted as a source of coronavirus infections not screened by surgical style masks.
The ViraCoat coating has been demonstrated to have profound antiviral properties against not just SARS-COV-2 (including all new variants, such as delta variant) but also against influenza (H1N1 seasonal flu strain) and bacteria (E. coli). The entire surface of the mask is covered, including the straps. The masks are a distinctive green colour to easily differentiate from standard PPE.

Jon Chadwick, CEO of ViraCoat's parent company ViraCorp, said: 'We are delighted that we have achieved ISO 18184 certification, which confirms our own testing through Lancaster University's world-class biomedical facilities. The level of protection we can offer healthcare workers and many others in hospitality, retail and travel, who risk exposure every day, is greatly enhanced and savings will result from fewer mask changes, plus we are reducing the number of masks ending up in landfill and our oceans'. 